\title{
BACTERIAL MENINGITIS MAKES A COMEBACK
}

$\mathbf{R}$ ecent reports from interstate and overseas have raised concerns about a resurgence of bacterial meningitis. In Victoria in 1989 there were 65 notified cases of meningococcal meningitis, with three deaths. This represented a threefold increase over the previous year. Outbreaks of meningococcal meningitis have been reported recently from Victoria ${ }^{1}$, Northern Territory ${ }^{2}$ and Western Australia.

Although meningococcal infection is notifiable in NSW, septicaemia and meningitis are not differentiated. Meningitis due to Haemophilus influenzae is not notifiable.

In May 1990 reports of two clusters of bacterial meningitis from the North Coast and the New England Regions prompted the following epidemiologic investigation of bacterial meningitis in NSW. The aims of the study were twofold:

\section{- to determine the true incidence of bacterial meningitis in NSW. \\ to assess the characteristics of three surveillance systems specifically for meningococcal meningitis: the existing infectious diseases notification system, active surveillance by Medical Officers of Health (MOHs), and the NSW Health Department Inpatient Statistics Collection (ISC).}

All separations from NSW hospitals are reported to the Health Department ISC. Specific conditions are coded according to ICD9-CM. We used this data source to identify hospital patients discharged from hospital between July 1, 1988 and June 30, 1990 with a diagnosis of bacterial meningitis (ICD9-CM 320) and meningococcal infection (ICD9-CM 036), assuming that people with these conditions would be admitted to public hospitals. We excluded records with a discharge diagnosis of neonatal meningitis or post-craniotomy meningitis as these conditions are not notifiable. Patients transferred between hospitals were counted according to the hospital of first admission. We attributed data from a hospital within any Area/Region to that Area Health Service/Region. No attempt was made to assess admission patterns across 'borders'.

Passive surveillance of infectious diseases occurs through medical practitioners notifying the Department of scheduled medical conditions. Although notification of meningococcal infections is required under the Public Health Act, we believe compliance with these provisions has been poor.

We initiated Statewide active surveillance of bacterial meningitis for the period January 1 to May 22, 1990 by asking $\mathrm{MOHs}$ from all 16 Health Areas and Regions to obtain details on all people admitted to hospitals with a primary diagnosis of bacterial meningitis or meningococcal disease. We requested data on age, sex, specific diagnosis, Aboriginality, date of hospital admission, date of separation and discharge status.

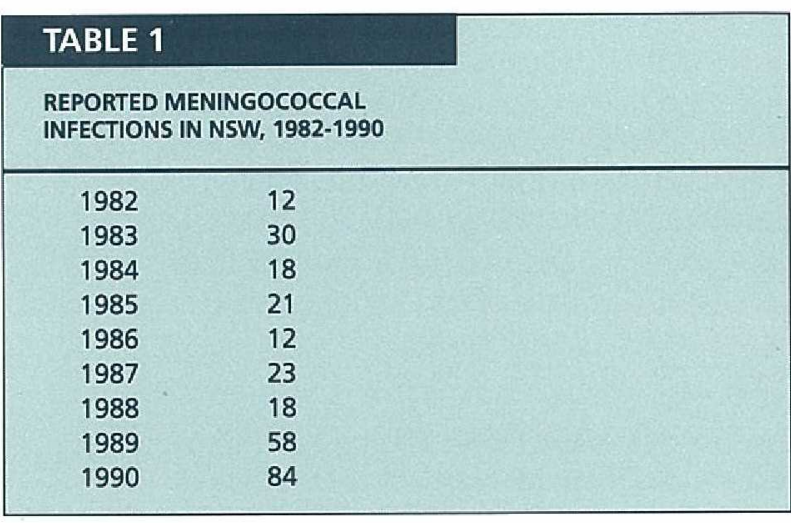

\section{TABLE 2}

BACTERIAL MENINGITIS IN NSW,

JAN 1-MAY 22, 1990,

BY AREA HEALTH SERVICE/REGION

OF NOTIFICATION

\begin{tabular}{|lcc|}
\hline & $\begin{array}{c}\text { Frequency } \\
\text { Rate }\end{array}$ & $\begin{array}{c}100,000 \\
\text { Population/Year }\end{array}$ \\
\hline Area Health Service & 4 & 3.1 \\
Central Sydney & 1 & 0.8 \\
Eastern Sydney & 6 & 2.1 \\
Northern Sydney & 3 & 1.5 \\
Southern Sydney & 8 & 3.4 \\
South West Sydney & 9 & 2.7 \\
Western Sector** & 3 & 3.6 \\
Central Coast & 11 & 5.9 \\
Hunter & 0 & 0.0 \\
Illawarra & & \\
Region & 11 & 8.3 \\
North Coast & 6 & 6.3 \\
New England & 3 & 5.5 \\
Orana \& Far West & 0 & 0.0 \\
Central West & 1 & 1.4 \\
South East & 7 & 7.2 \\
South West & 73 & \\
\hline Total & & \\
\hline ** Combined Western Sydney and Wentworth Area Health Services \\
\hline
\end{tabular}

As the active surveillance period was for 142 days, we computed annual incidence rates by multiplying rates by a factor of $365 / 142$. No attempt was made to interpret the non-linear distribution of bacterial meningitis with regard to seasonality.

The Chandra Sekar and Deming (CSD) method provides an estimate of cases not identified by either of two independent surveillance programs. We used this method to determine the number of cases not identified by the passive notification systems or the $\mathrm{MOH}$-initiated active surveillance system. 


\section{RESULTS \\ Incidence of bacterial meningitis}

In NSW from July 1, 1988 to June 30, 1989 there were 309 admissions for bacterial meningitis for a rate of 5.4/100,000 population. Of these, 71 were for meningococcal meningitis - a rate of 1.2/100,000.

Among children less than five years of age there were 35 admissions for meningococcal meningitis and 163 for other bacterial meningitis.

Between July 1, 1989 and June 30, 1990 there were 289 admissions for bacterial meningitis for a rate of 5.1/100,000 population. Of these admissions, 80 were for meningococcal meningitis - a rate of $1.4 / 100,000$.

\section{Surveillance of meningococcal meningitis}

The NSW infectious diseases database recorded the following numbers of notifications of meningococcal infections for 1982-90 (Table 1):

For January 1 to May 22, 1990 medical practitioners notified 14 patients with meningococcal infection through the existing infectious disease notification system.

All Health Areas and Regions responded to the request for hospital data. Active surveillance by the MOHs between January 1 and May 22 revealed 73 patients with bacterial meningitis. Of these, 30 were meningococcal (Tables 2, 3). Aboriginality was coded in only 11 per cent of records. Fifty-seven per cent of cases of meningococcal meningitis occurred in children under five years of age and of these, 88 per cent were aged less than two years (Table 4).

We could not link two patients notified by medical practitioners with patients detected through the active surveillance program. Using the CSD method we estimate that three patients with meningitis were not detected through either the active or the existing passive surveillance (Table 5).

The ISC identified 89 cases of bacterial meningitis for the surveillance period -26 with meningococcal meningitis.

\section{DISCUSSION}

\section{The incidence of bacterial meningitis}

In NSW the incidence of bacterial meningitis was $5.4 / 100,000$ for $1988 / 89$ and $5.1 / 100,000$ for $1989 / 90$. The rate for meningococcal meningitis for children under five years of age was calculated at $10.5 / 100,000$. The rate for $H$ influenzae meningitis for children under five was calculated at 13.6/100,000. These rates are all lower than similar rates in interstate and overseas reports.

Rates for the generic grouping 'bacterial meningitis' have been reported in a Scottish study as 16.9-17.8/100,0006. And in a recent article, case attack rates of $10-35 / 100,000$ are reported for epidemic periods of meningococcal disease in Australia?

Reported age-specific rates for $H$ influenzae meningitis in under five-year-olds for the United

States are $75-150 / 100,000$ and for Victoria, $58.5 / 100,000^{8}$.
There is seasonal variation in the incidence of meningococcal meningitis. Reported US experience noted that the highest incidence for bacterial meningitis occurs in winter and spring, with sporadic cases reported throughout the year?. In Victoria the peak incidence occurs in August and September, and the lowest incidence is in March and April?

As the active surveillance period corresponds to a trough period, extrapolation from our survey would underestimate the annual incidence in NSW (Table 2).

Surveillance of bacterial meningitis As only meningococcal meningitis is notifiable, comparison of the three surveillance systems is possible using this specific diagnosis as the index condition. Routine notification revealed 14 cases, active surveillance 30 , and hospital morbidity data 26. Extrapolation using CSD suggests a total of 35 cases of meningococcal meningitis during the surveillance period.

The discrepancy between the estimated 35 cases and those reported through surveillance mechanisms may be explained partly by the wide spectrum of clinical manifestations of this condition. Fulminant meningitis may cause death before hospital admission. Blood infection with meningococcus can occur with or without meningitis; the diagnosis of meningitis may be missed by clinicians ${ }^{10}$.

The existing notification system

Notification rates for bacterial meningitis have increased in 1989 and 1990, compared with the years 1982-1988 (Table 1), yet these rates are still lower than those reported in interstate and overseas studies ${ }^{678}$.

Meningococcal disease is notifiable by medical practitioners in NSW. Proposals for infectious disease notifications recommend that meningococcal disease continue to be notifiable with specification of septicaemia or meningitis; that Haemophilus

\section{TABLE 3 \\ PER CENT DISTRIBUTION OF PATIENTS WITH BACTERIAL MENINGITIS BY CAUSAL ORGANISM REPORTED BY MOHs, NSW JAN 1-MAY 22, 1990}

\begin{tabular}{|lcc|}
\hline Organism & No & Per cent \\
\hline Neisseria meningitidis & 30 & 41.1 \\
Haemophilus influenzae B & 22 & 30.1 \\
Streptococcus pneumoniae & 12 & 16.4 \\
Listeria monocytogenes & 4 & 5.5 \\
Staphylococcus aureus & 3 & 4.1 \\
Bacillus sp. & 1 & 1.4 \\
Klebsiella pneumoniae & 1 & 1.4 \\
\hline Total & 73 & 100.0 \\
\hline
\end{tabular}




\begin{tabular}{|c|c|c|}
\hline \multicolumn{3}{|c|}{$\begin{array}{l}\text { REPORTED BACTERIAL MENINGITIS } \\
\text { IN CHILDREN AGED LESS THAN } \\
\text { FIVE YEARS, NSW, JAN 1-MAY 22, } 1990\end{array}$} \\
\hline \multirow[t]{2}{*}{ Organism } & \multicolumn{2}{|c|}{ Age } \\
\hline & Under 2 & $2-4$ yrs \\
\hline Neisseria meningitidis & 15 & 2 \\
\hline Haemophilus influenzae B & 19 & 3 \\
\hline Streptococcus pneumoniae & 9 & 2 \\
\hline Bacillus sp. & 1 & 0 \\
\hline Total & 44 & 7 \\
\hline
\end{tabular}

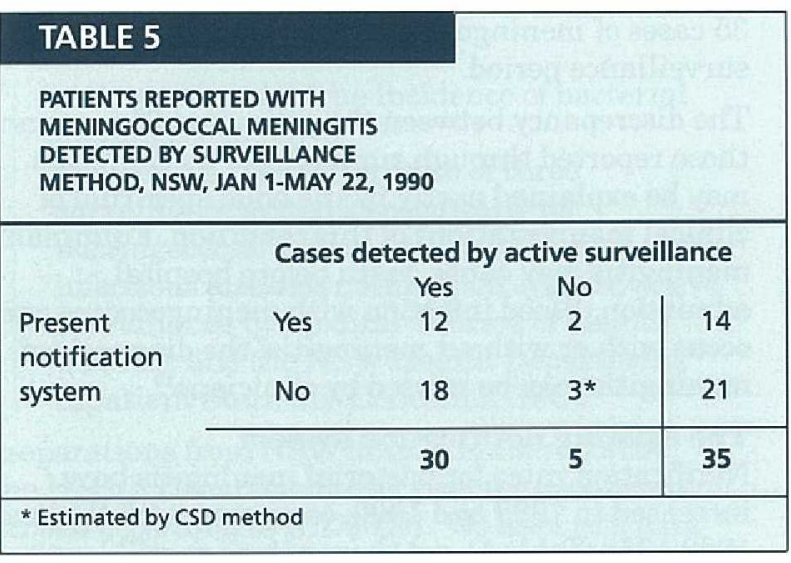

influenzae $B$ meningitis, septicaemia and epiglottitis become specifically notifiable; and that other forms of bacterial meningitis remain non-notifiable. Both laboratories and Chief Executive Officers/Regional Directors (or their delegate) would be asked to notify these conditions.

\section{Active surveillance by MOHs}

Using ISC as the 'gold standard', the sensitivity of active notification was 82 per cent (73/89).

The active surveillance project provided the most accurate information in terms of identifying cases in a timely manner. But the study was labour-intensive and therefore not ideal as a routine surveillance method.

\section{Inpatient Statistics Collection}

The ISC has no place to play in monitoring outbreaks of bacterial meningitis - cases are registered only on separation, not admission. Even if ISC were available 'on-line' Public Health Units would be alerted to an outbreak of meningitis only when treatment had been completed.

The discrepancy between the cases of meningococcal meningitis identified by $\mathrm{MOHs}(30)$ and those by the ISC (26) may be because MOH cases were admissions, while ISC reports on separations. Cases may have been admitted within the surveillance period but discharged after June 30, 1990, when the present collection was closed.
The poor coding of Aboriginality precludes analysis of this variable. It is the experience of the NSW Hospital Morbidity Collection that Aboriginality is coded in no more than 33 per cent of records (P Williams, personal communication). There is reason to believe the epidemiology of bacterial meningitis differs among Aborigines and non-Aborigines ${ }^{2}$.

The utility of hospital separation data is limited by delays of more than four months between close of collection period and availability of the information.

\section{RECOMMENDATIONS}

- PHU staff should develop improved surveillance strategies for detecting meningitis through better communication between Unit staff and health professionals in the Area Health Service/Region. Medical officers in hospitals could notify all cases of bacterial meningitis by telephone to the $\mathrm{MOH}$ for their Area Health Service/Region to initiate contact tracing and assess the need for prophylactic antibiotics.

- PHUs should investigate reasons for medical practitioners failing to notify meningitis. Where specific cases are identified through active surveillance or ISC, the treating practitioner would be contacted by the PHU staff, asked to submit a formal notification and asked the reasons for non-notification.

口 Epidemiology Branch should use ISC data to monitor the completeness of doctor- and laboratory-based notifications of meningitis.

- Laboratories should be asked to include the subtype of $\mathrm{N}$. meningitidis with their notifications. This is important to motivate the appropriate public health response; the meningococcal vaccine currently available is not recommended for use against type $\mathrm{B}^{\mathrm{T}}$.

Michael Levy, Wendy Manning, George Rubin

Epidemiology and Health Services Evaluation Branch NSW Health Department

1. Clements DA, Gilbert L. Increase in meningococcal infections detected at the Royal Children's Hospital, Melbourne. Communicable Diseases Intelligence 1990;8:4-7.

2. Meningococcal meningitis, Victoria and Central Australia. Communicable Diseases Intelligence 1989;21:3-4.

3. Watson C, Gardner V. A cluster of cases of group C meningococcal infection in Katanning, Western Australia. Communicable Diseases Intelligence 1990;5:4-6.

4. Shryock, HS and Siegal, JS (eds). Some methods of estimation for statistically underdeveloped areas. Methods and materials of demography. Academic Press, Orlando, Fla, 1974

5. NSW Health Department. Inpatient Statistics 1988/89.

6. Carter PE, Barclay SM, Galloway WH, Cole GF. Changes in bacterial meningitis. Arch Dis Child 1990;65:495-498.

7. Gilbert GL. Meningococcal infections: 1990 . Med J Aust 1990;153:507-508.

8. Gilbert GL, Clements A, Broughton SJ. Haemophilus influenzae type b infections in Victoria, Australia, 1985 to 1987. Ped Infect Dis J $1990 ; 9: 252-257$.

9. Benenson AS (ed), Control of Communicable Diseases in Man. 14th edition. American Public Health Association. Washington DC. 1985.

10. Mandell GL, Douglas RG, Bennett JE. Principles and Practice of Infectious Diseases. 3rd Edition. Churchill Livingstone. New York. 1990. 